\title{
COUNSELLING CHANGES IN SEXUAL FUNCTIONING FOR WOMEN WITH BREAST CANCER
}

\author{
LOHSY \\ Department of Rehabilitation Medicine, Faculty of Medicine, University of Malaya, Kuala Lumpur, Malaysia
}

\section{ABSTRACT:}

\begin{abstract}
As women with breast cancer are living longer, issues beyond survivorship like the much neglected sexual functioning and issue of quality of life have become increasingly important. Experiences of significant alterations in sexual functioning need to be addressed. However, these sexual issues are often not acknowledged in our traditional medical model of health care delivery. This paper briefly reviews the changes in sexual functioning after a diagnosis of breast cancer, with implication for clinical practice and medical curicula. (JUMMEC 2010; 13 (1): 33-37)
\end{abstract}

KEYWORDS: sexuality, oncology, counseling

\section{Introduction}

With over 100 years of research, it is now well known that breast cancer is a disease driven by hormones, genetics (sex, body compositions, genes), and lifestyle (1). Studies showed that there is a rapid increase in incidence rates of breast cancer before menopause (ages 40-50) and then a decline in rates (2). Women are now living longer with breast cancer, and may be dealing with numerous intimacy-, relationship-, and sexuality-related issues, including those related to reproduction (3), especially so for younger women (4, 5). The prevalence age of breast cancer onset amongst Malaysian women are generally younger at 40-50 years compared to developed countries.

Sex plays an important role, beside its basic function for procreation, in enhancing interpersonal relationships, and building a more intimate, meaningful bond. Managing the medical tasks, and the modifiable factors (like diet, weight, physical activity) are important $(2,6$, 7-10), but these should be complemented with patient self management of emotional tasks as well as role and relationships. American Cancer Society found that cancer survivors were not pursuing healthy lifestyles (11), and equally important, they need to pursue happy lifestyle behaviors. In fact, evidence shows that having a general feeling of happiness and optimism has a "protective effect" on the etiology of breast cancer (OR-0.75, 95\% Cl:0.64-0.86) (12). In short, issues of sex and sexuality are integral components of human behavior, adding romance, enjoyment and enhancing the quality of life of women. However, in the traditional
Asian clinics, sexuality is still neglected, and/or takes a back seat to cancer treatment and survival issues in people with cancer. Even in the more developed Asian countries, like Japan, a study on breast cancer patients $(n=102)$ found that discussing body image and sexuality were disregarded in therapeutic decisionmaking situations (13).

\section{Cancer treatment and altered sexual functioning}

Normal aging, by itself can greatly impair the sexual functioning of humans. Sexuality amongst men have gained a bit more attention where hypothetical surveys even suggest that men express a willingness to trade away survival time just so they can preserve or improve their sexual function (Singer PA, Tasch ES, Stocking C, et al 1991). Studies show an increase incidence of erectile dysfunction in men without cancer, up to threefold between the fifth and eighth decades of life (Feldman HA, Goldstein I, Hatzichristou DG, et al , 1994), whilst one-third of older men report experiencing a significant impairment in sexual function (Litwin, 1999). Do women have the same significant concerns and issues of sexual functioning? Studies have

\footnotetext{
Correspondence:

Loh Siew Yim

Department of Rehabilitation Medicine

Faculty of Medicine, University of Malaya

50603 Kuala Lumpur, Malaysia

Email: syloh@um.edu.my
} 
documented that approximately $50 \%$ of women who have experienced breast or gynecologic malignancy have serious concerns regarding sexual functioning (14), whereby sexual complaints can occur in up to $90 \%$ of women with a history of a cancer diagnosis (15). It appears that a diagnosis of breast cancer have further compounded whatever negative impact of normal aging on sexual functioning of women. One study found that sexual functioning of women $(n=558)$ who received chemotherapy compared to those who did not, regardless of the type of surgery $(p<0.001)$ was significantly affected (16). Many women are distressed by treatment-related sexual function or fertility-related adverse effects of treatment, but they are reticent to bring up the topic of sexuality given their lack of experience and low self-confidence, especially among the younger women (17).

Specifically, breast reconstruction has been shown to be associated with loss of breast sensation (18), whilst both mastectomy (with or without reconstruction) and lumpectomy were associated with altered body image; potentially affecting sexuality $(18,19)$, a decrease in perceived sexual attractiveness and reduced sexual interest (20). Radiation therapy causes changes in breast sensation, fatigue or arm mobility. (21), whilst Tamoxifen therapy is linked to pain, discomfort and vaginal tightness during intercourse (22). Women undergoing chemotherapy have experienced ovarian failure, hormonal changes, menstrual cycle disruption, amenorrhea, vaginal dryness and atrophy, and decreased sexual arousal and desire (23).

\section{The issues of sexual dysfunction amongst women}

In four focus groups conducted with Malaysian women living with breast cancer $(n=39)$, a rising theme was on the neglect of sexuality issues (24). In one of the groups, the women were quite open about their intimate relationships with their husband. This dispels the notion that Malaysian women in general are reluctant to talk about their private lives. However, as this was not typical in all groups, the use of focus groups may have led to their openness regarding the topic. These women's expressions negate the current belief that patients do not want to discuss about sexual issues. Sexuality and intimacy were two main role-related themes that emerged consistent as an unmet need across the groups. In general, the women felt that their intimate relationships were affected to some extent, but they felt the main reason was 'within themselves' rather than with their spouse whom they reported as being 'encouragingly supportive'. Some women seek clarifications and asked, 'Can we still have sex?' - as if sex after breast cancer will bring about detrimental consequences either on themselves or their spouse. In fact, one spouse of the informant came forth with his query on the toxicity of chemotherapy during sexual act of intimacy. While some utterances from the women may seem to be exaggerated, this issue of sexual concerns does seem to weigh heavily on both the survivors and their spouse's minds. Myths surrounding the issues of intimacy, chemotherapy and sexuality were not uncommon across the groups. Factors like age and side effects of hormonal treatment causing dryness; (including myths that too much excitement can trigger the cancer cells, and toxicity of chemotherapy can 'travel' to their spouse during sexual intercourse) were revealed (24). Acute or chronic sexual function problems resulting from treatments such as mastectomy, lumpectomy, radiation, and chemotherapy are not uncommon. The excerpt below highlights some examples of common myths related to chemotherapy and cancer.

"My husband believed that with chemotherapy, I have the toxins all over my body, so it's better not to have it (sexual intercourse)".

"I heard that cancer patient cannot have too much sex because I heard that sexual excitement can lead to recurrence, and I want to know if it's true or not?"

Cancer is a debilitating illness. It robs years from life and life from years because it traumatizes and detracts confidence, self-image, feelings of worth and pride, and the sense of normalcy from the survivors' daily functioning. One study examining the sexual problems of women below 50 years $(n=209)$, revealed specific problems in four areas (lack of interest in sexual activity, difficulty in becoming aroused, difficulty relaxing and enjoying sex, and difficulty achieving orgasm), with a lack of interest being the main challenge (25). These needs are critical but have been ignored in our traditional medical model care delivery system. Sexual functioning, like other functioning, needs to be viewed as fundamental to health and quality of life. Thus, management of cancer care must be emphasized as incomplete without full attention to 
the women's personal responses and experiences to illness, including sexual function. However, literature shows that hormonal and pharmacological therapies have been marketed to treat sexual desire and arousal disorders, and many if not none have been approved by the FDA nor have any been proven effective by clinical trials (26)

\section{Counseling Sexual Issues With women}

Psycho-behavioral intervention is a key part of the comprehensive sexual dysfunction treatment schema. In order for it to be comprehensive, an educational program that fosters open discussions regarding concern is a start. Healthcare professionals are often too embarrassed or feel ill-equipped to discuss sexual issues and patients are too embarrassed to ask (27). These issues of sexual functioning are often viewed as a difficult issue to handle when caring for patients. The topic is often absent from the curricula of most medical and health schools, as well as residency programs and fellowships program. This may explain why many healthcare professionals feel ill-equipped to address issues of sexuality during the course of routine health care and in the management of women with cancer. Any changes, for it to take effect, must target at the policy levels and to ensure health intervention are sufficiently broad based to address the vast needs (including addressing issues related to cultural myths) of the service users. It is especially pertinent to discuss sexuality issues with women - at before, during, and after cancer treatment. Therapists and health professionals in the oncology community need to sharpen their diagnostic and therapeutic skills in this area of sexuality counseling.

Counseling with survivors of breast cancer may start with the broad range of sexual function concerns that survivors as a group may experience, including fear and myths, genital pain, lack of lubrication, satisfaction, arousal, and desire, and then ask if the survivor has questions about any of these or would like information or referrals for additional information. Strategies like local non-medicated, nonhormonal vaginal moisturizers including vitamin $E$ suppositories agents, used two to three times weekly, can provide alternative relief for the symptoms of vaginal atrophy by maintaining the elasticity and pliability of the vaginal mucosal lining (28). Sexual function, body image, and relationship problems experienced by women due to breast cancer and cancer treatments may be addressed via individual and interpersonal counseling (29). A sensitive, openminded, and forthright attitude about issues of sexual functioning is timely when caring for women with breast cancer. Sexual counseling helps normalize the experience of sexual problems after cancer treatment, and can be effectively conducted in group work and/or further one-to-one intervention if needed. Conscious effort must be made to allow patients a safe environment to vent their fears, and provide the reassurance needed. A sexual psycho educational program in an oncology setting is necessary to provide comprehensive care to the patient. There is much to learn from the field of counseling which is deeply rooted in social-humanistic sciences. Overall, a bio-psychosocial model of care, in place of the stifle, traditional-hierarchical medical model of care can provide the foundation for addressing the much neglected aspect of sexual functioning amongst patients.

\section{Conclusion}

Breast cancer affects quantity and quality of life and every aspect of functioning, including sexual influences, the ultimate goal being to facilitate the readjustment of women towards independent functioning and meaningful living despite a diagnosis of breast cancer. In addressing the unmet sexual needs of women and providing counseling about changes in sexual functions, as well as therapeutic tips to enhance sexuality, healthcare professionals must be aware that they are treating the patient as a whole and not just the cancer.

\section{Acknowledgements}

The paper draws from the phase 1- qualitative study on the SAMA project funded by MAKNA and University Malaya Fundamental grant. The authors expressed deep gratitude to women who came forward to participate in the focus groups

\section{References}

1. Washbrook, E. Risk factors and epidemiology of breast cancer. Women's Health Medicine 2006; 3(1): 8-14. 
2. Bray F, McCarron P, Parkin D. The changing global patterns of female breast cancer incidence and mortality. Breast Cancer Research 2004; 6(6): 229239.

3. Walsh S, Manuel J, Avis N. The impact of breast cancer on younger women's relationships with their partner and children. Fam Syst Health 2005; 23: 80-93.

4. Avis N, Crawford S, Manuel J. Psychosocial problems among younger women with breast cancer. PsychoOncology 2004; 13(5): 295-308.

5. Bakewell RT, V DL. Sexual dysfunction related to the treatment of young women with breast cancer. Clin J Oncol Nurs 2005; 9: 697-702.

6. Stuart $\mathrm{K}$, et al. Life after breast cancer. Australian Family Physician 2006; 35(April 2006): 177-258.

7. Rock C, Demark-Wahnefried W. Can lifestyle modification increase survival in women diagnosed with breast cancer? J Nutrition 2002; 132(11): 3504 S.

8. Goodwin P, Boyd N. Body size and BC prognosis: A critical review of the evidence. Breast cancer Res Treat 1990; 16: 205-215.

9. Ziegler R, HooverR. Migration patterns and Breast cancer risk in Asian American woman. J Nat Cancer Institute 1993; 85: 1819-1827.

10. Deapen D, et al. Rapidly Rising Breast Cancer Incidence Rates Among Asian-American Women. Intl J Cancer 2002; 99 (3): 747-750.

11. Blanchard C, Courneya K, Stein K. American Cancer Society's SCS-II. Cancer survivors'adherence to lifestyle behavior recommendations and associations with health-related quality of life. $J$ Clin Oncol 2008; 26(13): 2198-2204.

12. Peled $R$, et al. Breast cancer, psychological distress and life events among young women. BMC Cancer 2008; 8(1): 245.

13. Adachi $K$, et al. Psychosocial factors affecting the therapeutic decision-making and postoperative mood states in Japanese breast cancer patients who underwent various types of surgery: body image and sexuality. Jpn J Clin Oncol 2007: hym041.
14. Andersen B. How cancer affects sexual functioning. Oncology (Huntingt.) 1990; 4: 81-94.

15. Anderson B, Woods X, Copeland L. Sexual selfschema and sexual morbidity among gynecologic cancer survivors. J Consult Clin Psychol 1997; 65: 221-229.

16. Ganz P, et al. Quality of life at the end of primary treatment of breast cancer: first results from the moving beyond cancer randomized trial. I Natl Cancer Inst 2004; 96(5):376-387.

17. Wenzel $L$, et al. Age-related differences in the quality of life of breast carcinoma patients after treatment. Cancer 1999; 86: 1768-1774.

18. Wilmoth M, Ross J. Women's perception: breast cancer treatment and sexuality. Cancer Pract 1997; 5: 353-359.

19. Schover L, Yetman R, Tuason L. Partial mastectomy and breast reconstruction: A comparison of their effects on psychosocial adjustment, body image, and sexuality. Cancer 1995; 75: 54-64.

20. Al-Ghazal S, Fallowfield L, Blamey R. Comparison of psychological aspects and patient satisfaction following breast conserving surgery, simple mastectomy and breast reconstruction. Eur $J$ Cancer 2000; 36: 1938-1943.

21. Bakewell R, Volker D. Sexual dysfunction related to the treatment of young women with breast cancer. Clin J Oncol Nurs 2005; 9: 697-702.

22. Mortimer J, et al. Effect of tamoxifen on sexual functioning in patients with breast cancer. $J$ Clin Oncol 1999; 17: 1488-1492.

23. Knobf $M$. The menopausal symptom experience in young mid-life women with breast cancer. Cancer Nurs 2001, 24: 201-210.

24. Loh SY, et al. Perceived barriers to self management in Malaysian women. AP J Pub Health 2007; 19(3): 53-57.

25. Burwell $S$, et al. Sexual Problems in Younger Women after Breast Cancer Surgery. J Clin Oncol 2006; 24: 2815-1821. 
26. Manson J, et al. Estrogen plus progestin and the risk of coronary heart disease. N Engl J Med 2002; 239 (6): 523-543.

27. Turns D. Psychosocial issues: pelvic exenterative surgery. J Surg Oncol 2001; 76:224-236.

28. Amsterdam A, Krychman M. Sexual function in gynecologic cancer survivors. Expert Rev Obstet Gynecol 2008; 3(3): 331-337.
29. Lev E. Counseling women with breast cancer using principles developed by Albert Bandura. Persp Psych Care 2000; 36(4): 131-138. 